ARTICLE

\title{
Grb2 monomer-dimer equilibrium determines normal versus oncogenic function
}

\author{
Zamal Ahmed ${ }^{1,2}$, Zahra Timsah"1,3,*, Kin M. Suen ${ }^{1,3, \star}$, Nathan P. Cook ${ }^{4, \dagger}$, Gilbert R. Lee IV ${ }^{2}$, Chi-Chuan Lin ${ }^{1,3}$, \\ Mihai Gagea ${ }^{5}$, Angel A. Marti ${ }^{4}$ \& John E. Ladbury ${ }^{1,2,3}$
}

The adaptor protein growth factor receptor-bound protein 2 (Grb2) is ubiquitously expressed in eukaryotic cells and involved in a multitude of intracellular protein interactions. Grb2 plays a pivotal role in tyrosine kinase-mediated signal transduction including linking receptor tyrosine kinases to the Ras/mitogen-activated protein (MAP) kinase pathway, which is implicated in oncogenic outcome. Grb2 exists in a constitutive equilibrium between monomeric and dimeric states. Here we show that only monomeric Grb2 is capable of binding to SOS and upregulating MAP kinase signalling and that the dimeric state is inhibitory to this process. Phosphorylation of tyrosine 160 (Y160) on Grb2, or binding of a tyrosylphosphate-containing ligand to the $\mathrm{SH} 2$ domain of $\mathrm{Grb} 2$, results in dimer dissociation. Phosphorylation of $\mathrm{Y} 160$ on Grb2 is readily detectable in the malignant forms of human prostate, colon and breast cancers. The self-association/dissociation of Grb2 represents a switch that regulates MAP kinase activity and hence controls cancer progression.

\footnotetext{
${ }^{1}$ Department of Biochemistry and Molecular Biology, University of Texas, M.D. Anderson Cancer Center, Unit 1000, 1515 Holcombe Boulevard, Houston, Texas 77030, USA. ${ }^{2}$ Center for Biomolecular Structure and Function, University of Texas, M.D. Anderson Cancer Center, Unit 1000,1515 Holcombe Boulevard, Houston, Texas 77030, USA. ${ }^{3}$ School of Molecular and Cellular Biology, University of Leeds, Leeds LS2 9JT, UK. ${ }^{4}$ Department of Chemistry, Rice University, Houston, Texas 77005, USA. ${ }^{5}$ Department of Veterinary Medicine and Surgery, University of Texas, M.D. Anderson Cancer Center, Unit 63, 1515 Holcombe Boulevard, Houston, Texas 77030, USA. * These authors contributed equally to this work. †Present address: Laboratory of Cellular Dynamics, Max Planck Institute for Biophysical Chemistry, 37077 Göttingen, Germany. Correspondence and requests for materials should be addressed to J.E.L. (email: j.e.ladbury@leeds.ac.uk).
} 
G rowth factor receptor-bound protein 2 (Grb2) is an adaptor protein with no intrinsic enzymatic activity which is ubiquitously expressed in all eukaryotic cells. It was originally discovered as epidermal growth factor receptor (EGFR) interacting protein ${ }^{1}$ but is now known to form complexes with a wide variety of cellular proteins including, but not limited to, protein tyrosine kinases, receptor tyrosine kinases, phosphatases, adaptors and intracellular scaffolds ${ }^{2-7}$. Grb2 has a modular architecture consisting of a central Src homology 2 (SH2) domain flanked by two Src homology 3 (SH3) domains. The N-terminal SH3 (N-SH3) has been reported to be constitutively associated with the mammalian homologue of the drosophila protein son-of-sevenless (SOS), a guanine nucleotide exchange factor (GEF) that acts on the Ras subfamily of small GTPases ${ }^{8}$. Under non-stimulatory conditions the Grb2-SOS complex resides in the cytoplasm. However, following receptor activation phosphorylated receptor tyrosine kinases (RTKs) and RTKdocking proteins such as Shc, IRS1-4 and FRS2/3 serve as recognition sites for the Grb2-SH2 domain ${ }^{6,9}$. As a result SOS is recruited to the plasma membrane leading to the activation of mitogen-activated protein kinase (MAPK) signalling cascade ${ }^{10,11}$.

Mechanisms through which control of signal transduction is exerted are vital in the regulation of cell response and function as well as in disease. With this in mind, two important features of Grb2 function have been reported, but their functional roles have not been fully elucidated: (1) Grb2 exhibits a monomer-dimer equilibrium ${ }^{12-14}$ and (2) tyrosine residues on Grb2 can be phosphorylated (for example by CD3 and CD4 (ref. 15), prolactin ${ }^{16}$ and vascular endothelial growth factor ${ }^{17}$ ). Furthermore, Grb2 is specifically phosphorylated on tyrosine residue 160 (Y160) by c-Src ${ }^{18}, \mathrm{BCR}^{\mathrm{A}} \mathrm{Abl}{ }^{19}$, fibroblast growth factor receptor 2 (FGFR2) ${ }^{20}$, NPM-ALK, TPR-Met and TEL-JAK2 (ref. ${ }^{21}$ ).

Here we resolve these elusive roles by demonstrating that Grb2 is only functional in MAPK signal transduction when it is in its monomeric form; and that dissociation of the constitutive dimer is facilitated by either phosphorylation of Y160 (pY160) or through the binding of a tyrosylphosphate-containing ligand. Therefore, rather than being a passive adaptor, Grb2 functions as a key control point in MAPK signalling. Our data also reveal the presence of high levels of pY160, the monomeric signalling active form of Grb2, in cancer tissue cells and suggest that this could be a marker for proliferative MAPK activation and resulting oncogenic outcome.

\section{Results}

Grb2 dimer is perturbed by phosphorylation of Y160. We sought to identify the factors regulating Grb2 monomer/dimer equilibrium which might define a switching mechanism for Grb2-mediated signal transduction. Since Y160 lies within the previously reported dimer interface and forms a hydrogen bond with E87 on the partner protomer ${ }^{13}$ (Fig. 1a), tyrosine kinase post-translational modification of Y160 could perturb dimerization through a repulsive charge or steric clash effect.

We generated a phosphorylation charge mimetic Grb2 mutant Y160E, designed such that the charge on the mutated glutamic acid residue repels the proximal E87 in the partner protomer and hence abrogates dimer formation (Fig. 1a). We also produced a mutant whereby two asparagine residues in the Grb2 dimer interface at positions 188 and 214 were mutated to aspartic acids (N188/214D). This mutant was unable to dimerize due to the juxtaposition of the two substituted negatively charged groups (Fig.1a). The N188/214D mutant provides a phosphorylationindependent dimerization-defective control protein to assess the physiological relevance of Y160 phosphorylation. The wild-type Grb2 ( $\left.{ }^{\mathrm{WT}} \mathrm{Grb} 2\right)$, Y160E ( ${ }^{\mathrm{Y} 160 \mathrm{E}} \mathrm{Grb} 2$ ) and N188/214D $\left({ }^{\mathrm{N} 188 / 214 \mathrm{D}} \mathrm{Grb2}\right)$ mutants were expressed and purified from bacteria. To confirm their stability we performed binding studies with known ligands (Supplementary Fig. 1). The monomerdimer equilibrium was measured by microscale thermophoresis (MST) after N-terminal labelling with Ato488. ${ }^{\mathrm{WT}} \mathrm{Grb} 2$ has an apparent dimerization constant $\left(K_{\mathrm{d}}\right)$ of $\sim 0.7 \mu \mathrm{M}$ (Fig.1b) consistent with the previously reported value ${ }^{12}$. The ${ }^{\mathrm{Y} 160 \mathrm{E}} \mathrm{Grb} 2$ and ${ }^{N 188 / 214 \mathrm{D}} \mathrm{Grb} 2$ on the other hand showed no monomerdimer transitions (Fig. 1c,d). In order to confirm the monomeric status of the two mutants we performed additional experiments using dynamic light scattering, analytical gel filtration, fluorescence anisotropy and time-resolved fluorescence anisotropy, native polyacrylamide gel electrophoresis (Supplementary Fig. 2). Under all conditions ${ }^{\mathrm{WT}} \mathrm{Grb} 2$ formed a concentrationdependent dimer whereas the two mutants were incapable of dimer formation.

Dimerization of ${ }^{\mathrm{WT}} \mathrm{Grb} 2,{ }^{\mathrm{Y} 160 \mathrm{E}} \mathrm{Grb} 2$ and ${ }^{\mathrm{N} 188 / 214 \mathrm{D}} \mathrm{Grb} 2$ was assessed in cells using fluorescence lifetime imaging microscopy (FLIM) to measure fluorescence resonance energy transfer (FRET) between two Grb2 protomers. Cyan fluorescence protein (CFP)-tagged Grb2 (CFP-Grb2) and red fluorescence protein (RFP)-tagged Grb2 (RFP-Grb2) were co-transfected into mammalian cells. Grb2 dimerization can only be seen in cells expressing the wild-type Grb2 (CFP_WT Grb2/RFP-WT Grb2) (Fig. 1e and Supplementary Fig. 3). Cells expressing ${ }^{\mathrm{Y} 160 \mathrm{E}} \mathrm{Grb} 2$ and ${ }^{\mathrm{N} 188 / 214 \mathrm{D}} \mathrm{Grb} 2$ show no evidence of dimerization.

To demonstrate that phosphorylation of the wild-type Grb2 disrupts dimerization, we devised a pseudo single molecule FLIM (smFLIM) experiment. Bacterially expressed and purified His-tagged ${ }^{\mathrm{WT}}$ Grb2 was N-terminally labelled with Atto488 and immobilized on Talon beads, while non-tagged ${ }^{\mathrm{WT}} \mathrm{Grb} 2$ was labelled with Atto550. After measurement of the reference lifetime of Atto488-WT $\mathrm{Grb} 2$, Atto550- ${ }^{\mathrm{WT}} \mathrm{Grb} 2$ was added and allowed to form a complex. The fluorescence lifetime population showed a distinct left shift to a shorter value indicating that FRET has occurred (Fig. 2). Incubation for one hour in the presence of purified FGFR2 cytoplasmic domain alone had no effect on the FRET lifetime between the Grb2 protomers. However a further hour of incubation in the presence of ATP and $\mathrm{MgCl}_{2}$ in order to phosphorylate Y160 on Grb2 (ref. 20) resulted in a shift of the average fluorescence lifetime towards the original reference lifetime for Atto488- ${ }^{\mathrm{WT}} \mathrm{Grb} 2$ alone (Fig. 2), demonstrating that the formation of the Grb2 dimer was abrogated by phosphorylation. These findings clearly demonstrate that the disruption of Atto488-Grb2/Atto550-Grb2 complex is FGFR2 activation dependent.

Tyrosylphosphorylated ligand results in dimer dissociation. From the reported crystal structure ${ }^{13}$ Y160 appears to form a hydrogen bond with E87 of the partner protomer (Fig. 1a). Since E87 lies within the conserved FLI/VRE motif of the SH2 domain (which is involved in recognition of phosphorylated tyrosine residues), the insertion of a tyrosylphosphate into the binding pocket on the SH2 domain might be expected to perturb the side chain position of E87 and disrupt the interaction between Y160 and E87. Using the previously described smFLIM method we determined that the addition of a tyrosylphosphopeptide based on the sequence of the known Grb2 ligand Shc (pShc) leads to dissociation of Grb2 dimers into the monomeric form (mGrb2) (Fig. 3). The addition of a proline-rich peptide derived from SOS which binds to the SH3 domains of Grb2 had no effect on dimerization on the surface of the Talon beads (Supplementary Fig. 4a). We confirmed this observation by measuring the monomer-dimer equilibrium of Grb2 using MST. Since Grb2 is 
a

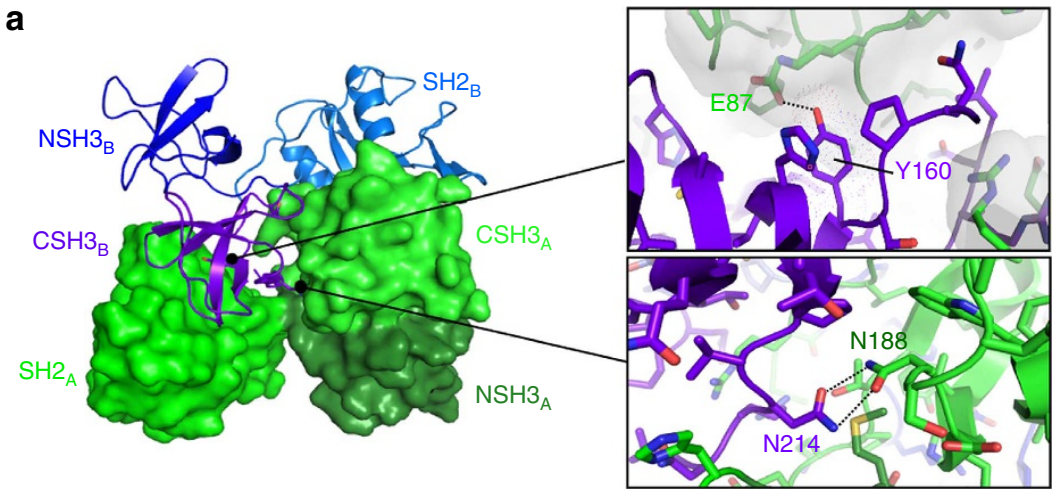

b

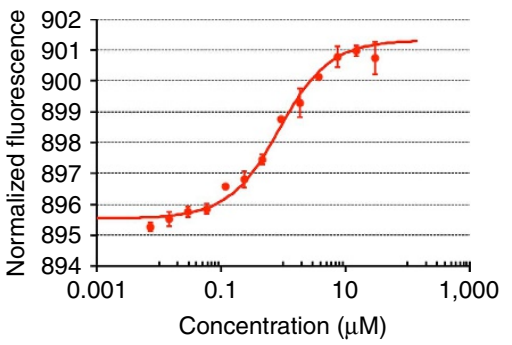

C

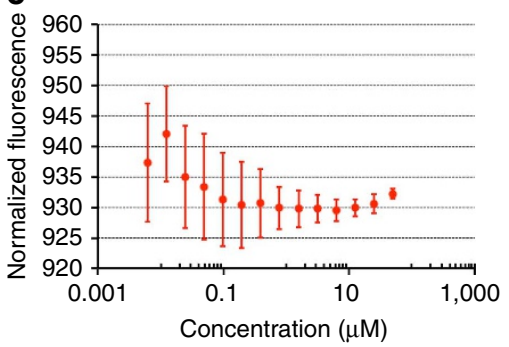

d

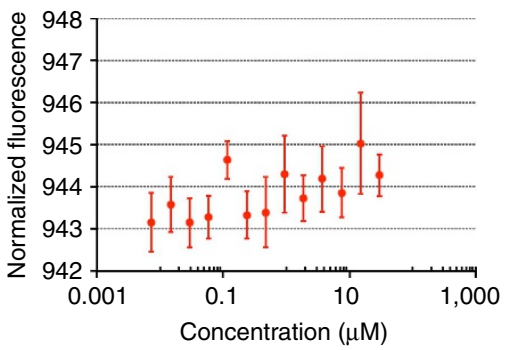

e

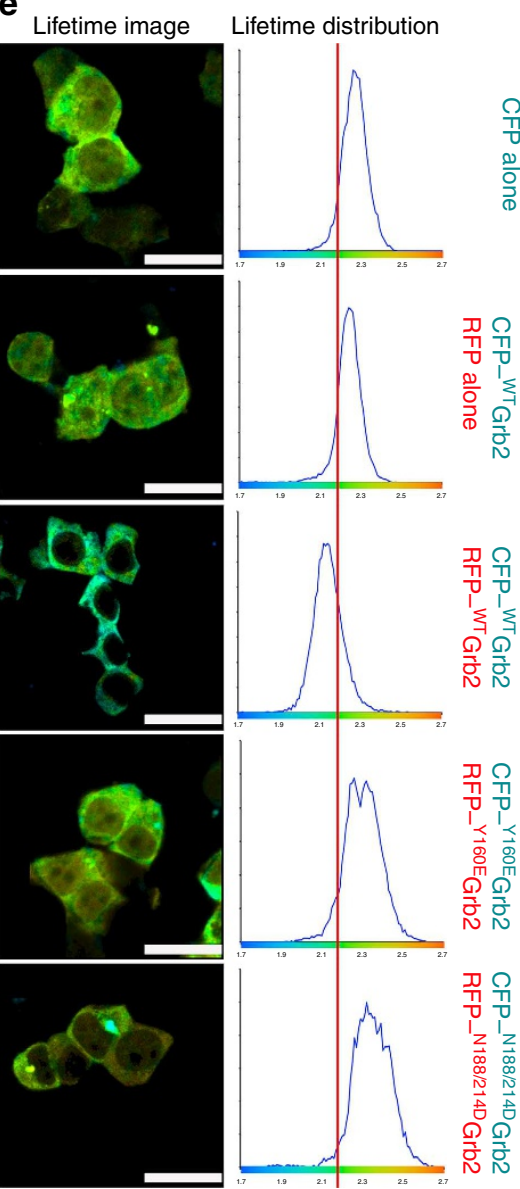

Figure 1 | In vitro and in vivo ${ }^{\mathbf{W}}$ Grb2 form dimers. (a) Homodimer with individual Grb2 protomers of the dimer depicted using surface (green) and ribbon (blue/purple) representations (derived from PDB 1GRI). Individual SH2 and SH3 domains of the protomers are differentially coloured and labelled. Zoomed box (top) - Y160 of one protomer (purple) is buried in the dimer interface, packing against the CSH3 domain and hydrogen bonding with E87 of the other protomer chain (green). Zoomed box (bottom)-hydrogen bonded Asn188 (green) and Asn214 (purple) in the dimer interface. (b) Grb2 dimerization measured by MST. Unlabelled ${ }^{\mathrm{WT}} \mathrm{Grb} 2$ protein $(7.3 \mathrm{nM}$ to $30 \mu \mathrm{M}$ ) was titrated into a fixed concentration of labelled Grb2 (100 nM). The data for thermophoresis was recorded at $20^{\circ} \mathrm{C}$ using the blue LED at $20 \%$ and IR-Laser at $40 \%$. The isotherm derived from the raw data and fitted according to the law of mass action to yield an apparent $K_{d}$ dimer of $0.76 \pm 0.20 \mu \mathrm{M}$. (c) The data points representing the MST results of the Y160E mutant, which show a scattered distribution and unsuitable for fitting. (d) The thermophoresis results of the N188/214D mutant, as with Y160E the data is unsuitable for fitting analysis. (e) FLIM of Grb2 dimerization in mammalian cells through FRET measurements. CFP- and RFP-tagged wild-type or the Grb2 mutants together with controls were co-transfected in HEK293T cells as indicated on the figure. The results show a clear shortening of the average lifetime distribution for fluorophore in cells expressing the WTGrb2. This is due to FRET between the CFP-Grb2 and RFP-Grb2. The Y160E and N188/214D mutants on the other hand show an average lifetime distribution comparable to the controls indicating no FRET. Scale bar, $25 \mu \mathrm{m}$.

monomeric, in the presence of a saturating concentration of pShc no apparent change in the oligomeric state of the protein was observed (Supplementary Fig. 4b). As expected similar experiments in the presence of the SOS1 peptide, Grb2 undergoes a dimer-to-monomer dissociation which has an identical apparent $K_{\mathrm{d}}(\sim 0.7 \mu \mathrm{M})$ to wild-type protein in the absence of peptide binding (Supplementary Fig. 4c). These results are consistent with previously reported NMR data where Grb2 


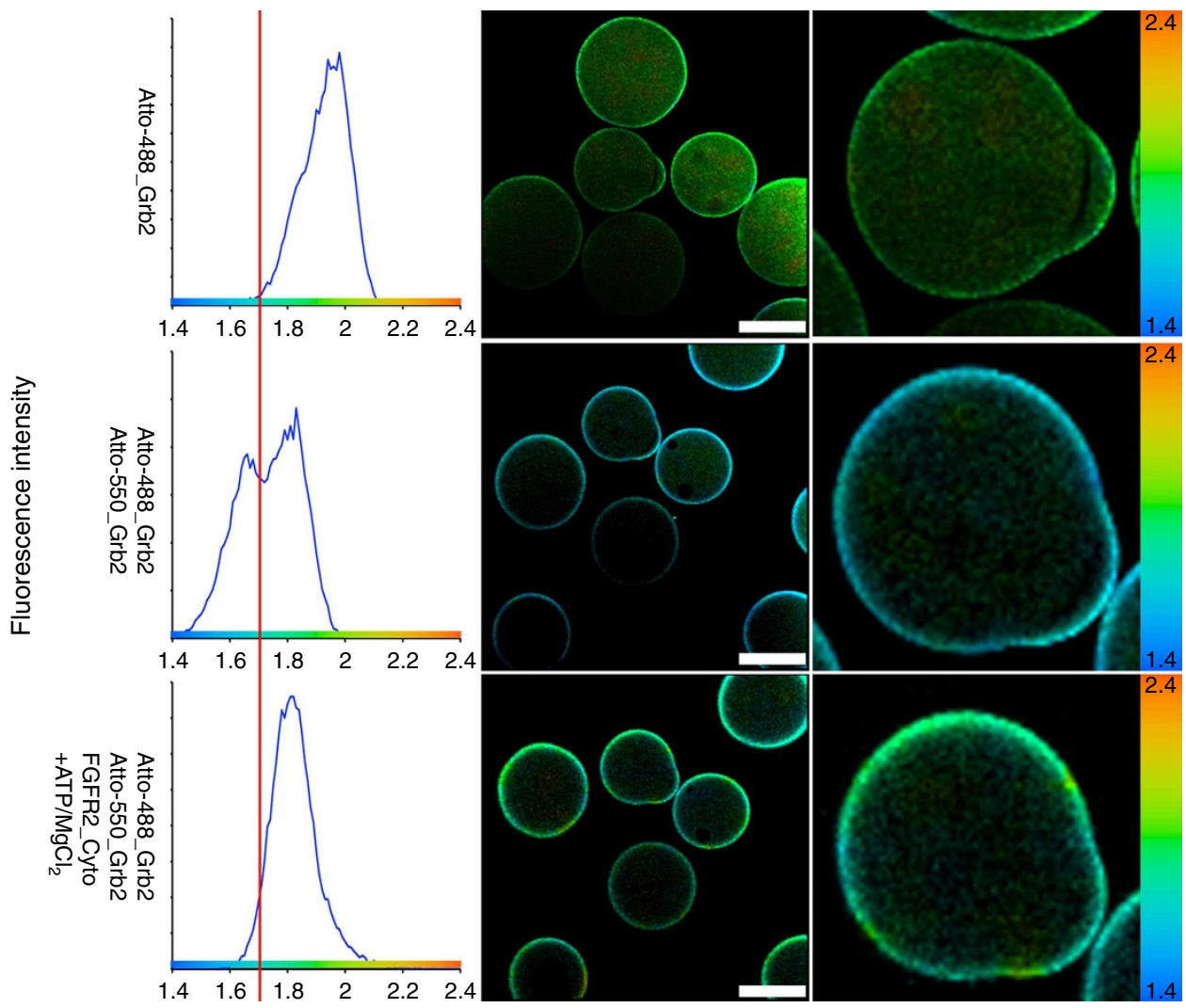

Figure 2 | Grb2 tyrosine phosphorylation disrupts dimerization. FRET between Atto488-Grb2 and Atto550-Grb2 measured with FLIM. The panels on the right show the distribution of the average fluorescence lifetime. The middle image is the representation of the measured lifetime as a false colour map. On the right hand side are zoomed images of a bead with a characteristic imperfection. The mean lifetime of Atto488-Grb2 is centred at $\sim 1.9 \mathrm{~ns}$. The addition of the acceptor Atto550-Grb2 leads to an apparent left shift in the average lifetime. This is clear from the appearance of a new peak at $\sim 1.6 \mathrm{~ns}$ as a result of FRET between Atto488-Grb2 and Atto550-Grb2. Addition of FGFR2 has no effect on the apparent average lifetime. However, further addition of ATP/ $\mathrm{MgCl}_{2}$ reversed the average lifetime to $1.9 \mathrm{~ns}$ providing evidence for the disruption of FRET and hence the disruption of dimerization on phosphorylation of Grb2. Scale bars, $50 \mu \mathrm{m}$.

was found to be monomeric in the presence of a phosphopeptide ${ }^{22}$.

Monomeric Grb2 is the signalling active form. We have shown two independent mechanisms of Grb2 dissociation, both dependent on tyrosine kinase activity. Both these mechanisms could play a fundamental role in Grb2-mediated intracellular signalling. Since Grb2 is known to bind to its downstream effector SOS1, we postulate that only mGrb2 elicits downstream effector function and hence the dimeric Grb2 (dGrb2) is incapable of signal transduction. We tested this hypothesis by investigating the downstream signalling effects of mGrb2 versus dGrb2 in mammalian cells. In cells overexpressing high concentrations the population of Grb2 is expected to be predominantly dGrb2, whilst the dimerization-defective Grb2 mutants will be monomeric regardless of their intracellular concentrations. Cells were transfected with ${ }^{\mathrm{WT}} \mathrm{Grb} 2$ and ${ }^{\mathrm{Y} 160 \mathrm{E}} \mathrm{Grb} 2$, and downstream MAPK signalling was compared. The results show that overexpression of ${ }^{\mathrm{WT}} \mathrm{Grb} 2$ leads to a significant decrease in FGF2- and EGF-induced extracellular signal-regulated kinases (ERK) phosphorylation. An equivalent level of dimerizationdefective mutant overexpression on the other hand retains ligandinduced ERK activity (Fig. 4a and Supplementary Fig. 5a). We also confirmed Grb2 concentration-dependent MAPK inhibition by serially increasing in the intracellular Grb2 protein concentration in cells. A direct correlation between increased Grb2 concentration and decreased MAPK was observed
(Supplementary Fig. $5 c-e$ ). Since the formation of the Grb2-SOS complex is pivotal for RTK-stimulated Ras/MAP kinase activation, we investigated whether the differential complex formation by dGrb2 versus mGrb2 with SOS1 could explain the observed suppression of ERK phosphorylation. Grb2 was affinity purified from cells and the resulting co-precipitating complex was analysed for SOS1 (Fig. 4b and Supplementary Fig. 5b). The results show that the monomeric ${ }^{\mathrm{Y} 160 \mathrm{E}} \mathrm{Grb} 2$ and ${ }^{{ }^{1188 / 214 D}} \mathrm{Grb} 2$ form stable complexes with SOS1 whereas the dimeric ${ }^{{ }^{\mathrm{TT}} \mathrm{Grb} 2}$ does not. As a control the immunoblot was re-probed with antiFRS2 antibody, which showed that both dGrb2 and $\mathrm{mGrb} 2$ were equally proficient in precipitating the upstream adaptor protein required for FGF receptor signalling.

The direct interaction of either dGrb2 or mGrb2 with SOS1 in cells was tested using FLIM. RFP-tagged ${ }^{\mathrm{WT}} \mathrm{Grb} 2$ and ${ }^{\mathrm{Y} 160 \mathrm{E}} \mathrm{Grb} 2$ were co-transfected with GFP-tagged SOS1 (GFP-SOS1). The results show that the monomeric Grb2 mutants form more stable interactions with SOS1 than ${ }^{\mathrm{WT}} \mathrm{Grb} 2$ (Supplementary Fig. 6). Together these results confirm that a monomeric Grb2 is necessary and sufficient to form the interaction with SOS1 and propagate the MAPK signalling pathway.

Monomeric Grb2 is associated with malignant cancers. Y160 is a target phosphorylation site for many receptor and non-receptor tyrosine kinases that are involved in cancer growth and metastasis ${ }^{21}$. This suggests dGrb2 may have an inhibitory role whilst $\mathrm{mGrb} 2$ promotes proliferative downstream signalling. 


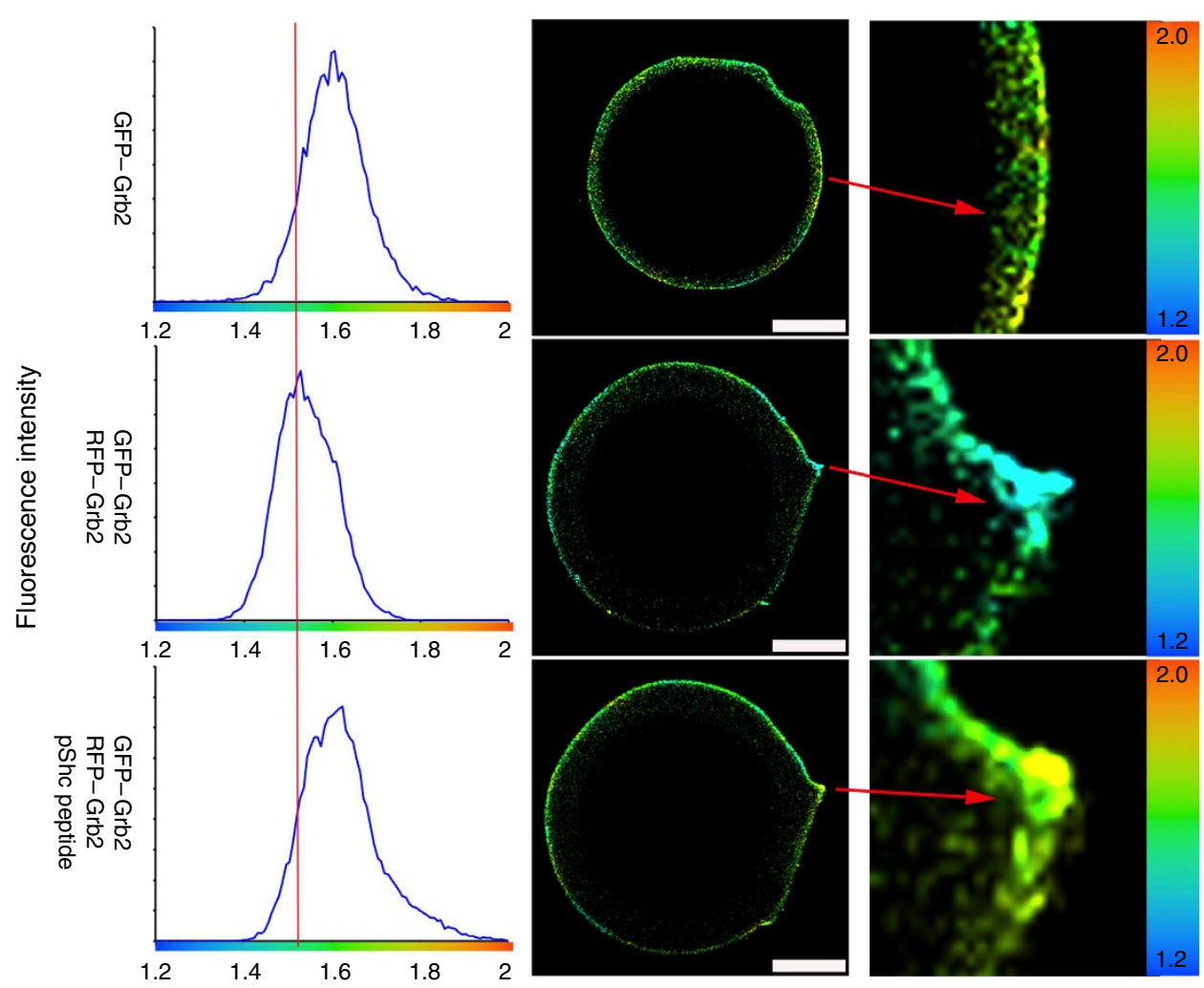

Figure 3 | Binding of phosphotyrosine by Grb2-SH2 domain leads to dissociation. smFLIM shows Grb2 dimerization is disrupted upon phospho-Shc peptide binding by its $\mathrm{SH} 2$ domain. GFP-tagged Grb2 immobilized on GFP-trap beads were imaged in $20 \mathrm{mM}$ Tris- $\mathrm{HCl}, 50 \mathrm{mM} \mathrm{NaCl}$ at pH 8.0 . The reference average lifetime in the absence of acceptor is $1.6 \mathrm{~ns}$. RFP-Grb2 was added and allowed to form complex for $1 \mathrm{~h}$ and the lifetime of GFP-Grb2 shortens as indicated by left-shifted peak with average lifetime centred on $1.5 \mathrm{~ns}$ clearly indicating that FRET has occurred. Phospho-Shc peptide (10 $\mu \mathrm{M}$ ) containing the pYxN motif was then added, allowed to equilibrate for an hour and the fluorescence lifetime was measured. Addition of the phospho-Shc peptide restores the lifetime to the control values obtained in the absence of acceptor. This clearly shows that the phospho-Shc peptide disrupts dimerization of Grb2. The zoomed image of the bead (right hand column) shows lifetime values mapped to a false colour image. The change in fluorescence lifetime as a function of colour is highly noticeable. The data presented here was consistently reproduced in three independent experiments. Scale bars, $10 \mu \mathrm{m}$.

Here we have shown that Y160 phosphorylation-dependent dissociation of dGrb2 is sufficient to induce upregulation of MAPK signalling (Fig. 4) which is associated with proliferation and metastasis $23-25$. Therefore, we would predict that in tyrosine kinase-driven cancers phosphorylation of Y160 and the resulting dissociation of Grb2 dimers would be capable of inducing cell proliferative and metastatic outcome. This would suggest that pY160 could be used as marker for elevated tyrosine kinasemediated ERK signalling in human cancers. We therefore generated a site-specific anti-pY160 antibody against pY160. This antibody was tested exhaustively and found to be suitable for detecting Y160-phosphorylated Grb2 in western blotting and in immunohistochemistry (IHC) analysis (Supplementary Fig. 7). We performed IHC analysis of a multi-tumour tissue array containing 40 human tumour samples from 27 different organs. The results show that Y160 was highly phosphorylated in a significant number of high-grade malignant tumours but not in the lower grade or non-malignant counterpart tumours or in corresponding normal tissue (Supplementary Fig. 8). Y160 phosphorylation was particularly prevalent in higher grade, late-stage malignant tumours of the colon and prostate (Fig. 5a-d). Therefore, we performed additional IHC analysis on 118 colon and 42 prostate cancer tissues with the anti-pY160 antibody, which shows a clear correlation between disease progression and Grb2 phosphorylation (Fig. 5e,f). Higher levels of Y160 phosphorylation were again clearly associated with higher-grade malignant tumours. In addition we performed protein microarray analysis on 55 human breast tumours with matched normal tissue using the pY160 antibody. The results revealed Y160 phosphorylation was absent in normal breast samples, however 12 out of 55 breast tumour samples were positive for pY160 (Supplementary Fig. 9a-c). To confirm the results of the protein microarray, we selected two positive breast tumour samples and the corresponding normal matched sample for western blotting analysis. The results show elevated Grb2 expression and phosphorylation, MAP kinase and tyrosine kinase activity in the tumour samples compared with the normal cohort. Furthermore, it was possible to assign the elevated pY160 level of one patient to increased EGFR expression and kinase activity (Supplementary Fig. 9d).

\section{Discussion}

The discovery of the adaptor protein Grb2 as receptor tyrosine kinase-interacting protein 20 years ago was a key point in defining RTK signal transduction ${ }^{26,27}$. Since then a plethora of Grb2-interacting proteins have been reported (http://stringdb.org). The interaction between Grb2 and SOS and the recruitment of the Grb2-SOS complex to the plasma membrane to activate the MAPK protein kinase cascade is a pivotal function of Grb2 in cells. It has been known for some time that Grb2 could form dimers, however it has been debated as to whether the dimeric state had any physiological relevance. Here we used both in vitro and in vivo studies to show 

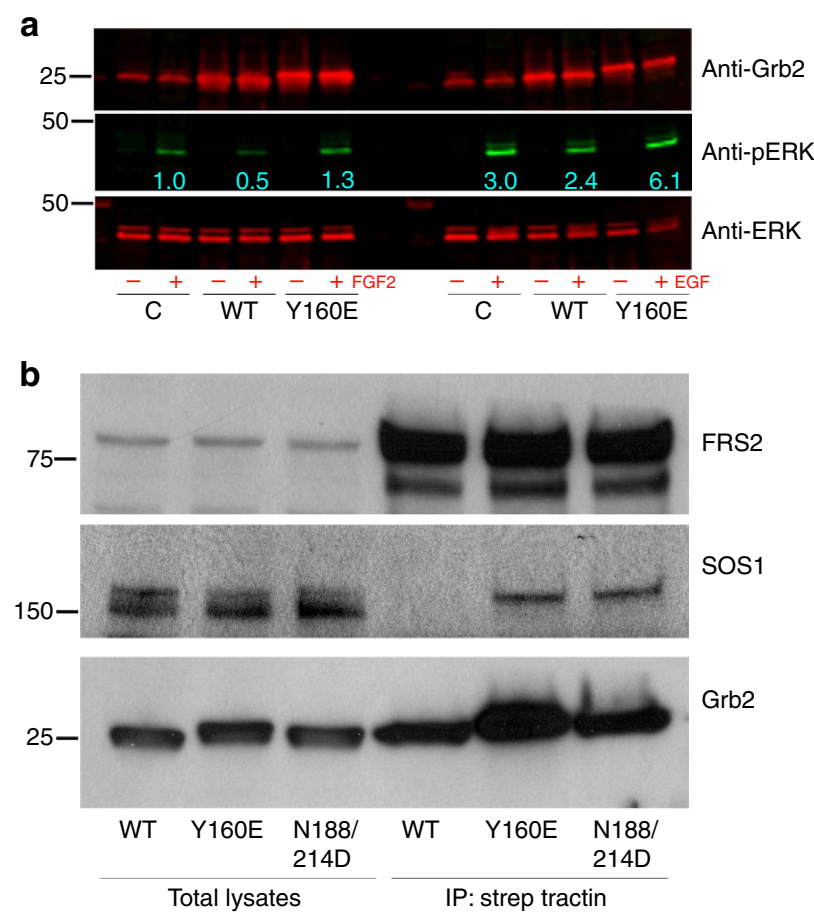

Figure 4 | Dimeric Grb2 inhibits while monomeric Grb2 promotes MAP kinase activity. (a) Stable HEK293T cells overexpressing FGFR2-GFP (as control, C) and strep-tagged wild-type Grb2 or the dimerizationdefective Y160E mutant were stimulated with $50 \mathrm{ng} / \mathrm{ml} \mathrm{FGF2} \mathrm{or} \mathrm{EGF} \mathrm{for} 15$ and $5 \mathrm{~min}$, respectively. Cell-lysates were analysed for phospho-ERK (pERK), total ERK and Grb2 expression levels using specific antibody and Odyssey infra-red imaging. (b) HEK293T cells overexpressing FGFR2-GFP with indicated strep-tagged Grb2 in serum were lysed and subjected to strep-tactin affinity purification. The resulting co-precipitated complexes along with input cell-lysates were analysed for SOS and FRS2 binding using respective antibody. The immunoblot was also re-probed for Grb2 as a loading control. The data presented are representative of three independent experiments.

concentration-dependent Grb2 dimerization (Fig. 1 and Supplementary Fig. 2). We have demonstrated that the monomer-dimer population can be perturbed by Y160 phosphorylation and the binding of a ligand to the $\mathrm{SH} 2$ domain of Grb2 (Figs 2,3). We addressed the issue of whether there is a functional consequence of disruption of the dimeric state in cells and demonstrated that the regulation of the selfassociation state of Grb2 is intimately related to the receptor tyrosine kinase activity. The monomeric form is the signallingcompetent active form whereas dimeric Grb2 is the inactive inhibitory form. Only monomeric Grb2 associates with SOS, while both monomeric and dimeric form can engage a phosphorylated tyrosine residue (Fig. 4b).

The change from dimeric-monomeric state in cells is strongly influenced by upregulation of kinase activity and the resultant phosphorylation of Grb2 itself or of cognate ligands. The requirement of Grb2 to dissociate from the dimeric state provides a further 'check-point' in MAPK signal transduction. Driving the dissociation of Grb2 through increased kinase activity can be reversed by phosphatases. We have previously reported that the tyrosine phosphatase Shp2 is a specific phosphatase for Grb2 (ref. 20).

Phosphorylation, however, is not the only mechanism for dGrb2 dissociation. Protein expression levels will also impact on this. This is reflected in downstream signalling, where overexpression of wild-type Grb2 increases the population of dimer and hence suppresses MAPK activation (Fig. 4a and Supplementary Fig. 5). However, overexpression of Grb2 coupled with Y160 phosphorylation leads to increased MAPK activation (Supplementary Fig. 9). Thus, initiation of MAPK signalling is modulated by several cellular conditions and fluctuations in these could have significant impact on cellular outcome.

Human cancer tissue analysis shows a significant increase in Grb2 phosphorylation in high-grade pre-metastatic tumours, further highlighting the importance of $\mathrm{mGrb} 2$ in signal transduction and cancer progression. Therefore, the mechanistic insight gained from this work might pave the way for developing novel strategies towards cancer therapy, based on stabilizing the formation of Grb2 dimers. One inventive way of achieving this might be based on a small molecule which could irreversibly crosslink the two monomers across the dimer interface. Together these results from human cancer patients confirm the importance of mGrb2 in intracellular signalling and suggest that pY160 could be used as a potential predictive biomarker in personalized cancer therapy.

\section{Methods}

Reagents. Anti-Grb2 (C23), anti-FRS2 and anti-SOS antibodies were obtained from Santa Cruz Biotechnology. Anti-phospho-Erk and anti-total Erk antibodies were from Cell Signaling. All antibodies were diluted to 1:1,000. Anti-pY160 antibody was raised against QPT(pY)VQALFDFDPQC peptide (GenScript). Phospho-EGFR peptide FLPVPE(pY)INQSVPKR, phospho-Shc peptide EEPPDHQ(pY)YN-DFPGK and SOS1 peptide PVPPPVPPRRRPEY were purchased from GenScript. The SomaPlex Protein Microarray of Human Breast Tumor \& Normal Tissue (PMA2-001-L), as well as the matching cell lysates were purchased from Protein Biotechnologies. Multi-organ tissue microarray slides (MUT951), colon cancer tissue array (COC1262) and prostate cancer tissue array (PRC481) were purchased from Pantomics.

Molecular cloning. Gene fragments encoding Grb2 were cloned into pECFP-C1 (Clontech) in the BamHI site to create the CFP-Grb2 construct. The monomeric RFP (mRFP) was cloned into the NheI/HindIII site of the pcDNA3.1 $(+)$ vector (Invitrogen). Grb2 was then subcloned into the BamHI/EcoRI sites to create the RFP-Grb2 construct. In both cases the fluorescent tag was placed at the $\mathrm{N}$-terminus of Grb2. For histidine-tagged protein expression and purification, Grb2 was cloned into BamHI/EcoRI site of a modified pET28a vector containing tobacco edge virus protease (TEV) cleavage site. The Y160E and N188/214D mutations were introduced into Grb2 by site-directed mutagenesis. Bacterial protein expression and purification were performed as described previously ${ }^{20,28,29}$. Strep-tagged Grb2 was created by fusing a WSHPQFEK sequence at the $\mathrm{N}$-terminus of the full-length Grb2. The PCR amplified DNA fragment was inserted into the HindIII/PmeI site of pcDNA6-myc/His C vector (Invitrogen). Bacterial GFP-Grb2 and RFP-Grb2 were created by cloning NdeI-SacI-BamHI(GFP or RFP)-HindIII-EcoRI-XhoI fragments from pEGFP-N1 or mRFPpcDNA3.1, respectively, into the NdeI and XhoI site of a pET28a vector. The fulllength Grb2 was then subcloned into the HindII/XhoI sites. Molecular cloning expression and purification of FGFR2 cytoplasmic domain in bacteria is described elsewhere $^{12}$.

Cell culture. HEK293T cells obtained from the American Type Culture Collection were maintained in Dulbecco's modified Eagle's high glucose medium (DMEM) supplemented with $10 \%$ (vol/vol) fetal bovine serum (FBS) and $1 \%$ antibiotic/ antimycotic (Lonza) in a humidified incubator with $10 \% \mathrm{CO}_{2}$ as described previously ${ }^{30-32}$. Stable HEK293T cells overexpressing GFP-tagged FGFR2 were produced as described previously ${ }^{30}$. Control and Grb2 knockdown cells were produced as described previously ${ }^{20}$. Mammalian cell transfection was performed in a six-well plate using Metafectin Pro (Biontx-USA) reagents according to the manufacturer's instructions. Cells were lysed in $50 \mathrm{mM}$ HEPES ( $\mathrm{pH} 7.5), 1 \%(\mathrm{v} / \mathrm{v})$ Igepal-C630, $1 \mathrm{mg} \mathrm{ml}^{-1}$ bacitracin, $1 \mathrm{mM}$ EDTA, $10 \mathrm{mM} \mathrm{NaF}, 1 \mathrm{mM}$ sodium orthovanadate, $10 \%$ (v/v) glycerol, $50 \mathrm{mM} \mathrm{NaCl}, 1 \mathrm{mM}$ PMSF and Protease Inhibitor Cocktail Set III (Calbiochem).

Immunoblotting. Immunoblotting was performed using the indicated antibodies. Blots were cropped such that at least one marker position was present. Uncropped full scans are shown in Supplementary Fig. 10.

Protein expression and purification. $6 \times$ Histidine-tagged constructs of FGFR2 and Grb2 were expressed and purified from E. coli as described in refs ${ }^{28,32}$. It should be noted that purified Grb2 is inherently unstable as a monomer and can be affected by inter-molecular disulphide bond formation. 
a

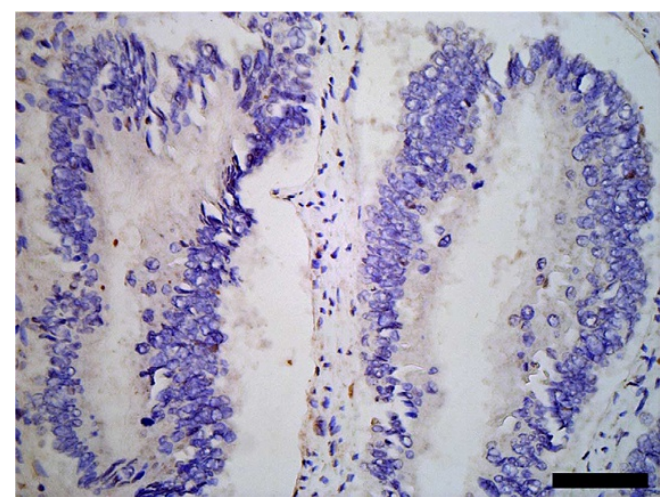

C

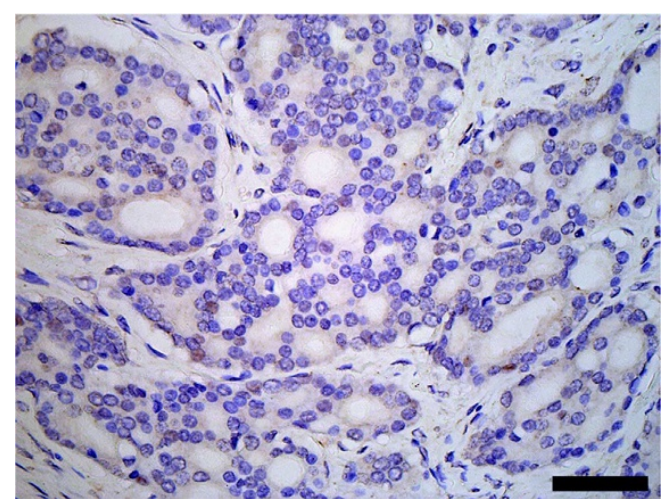

e

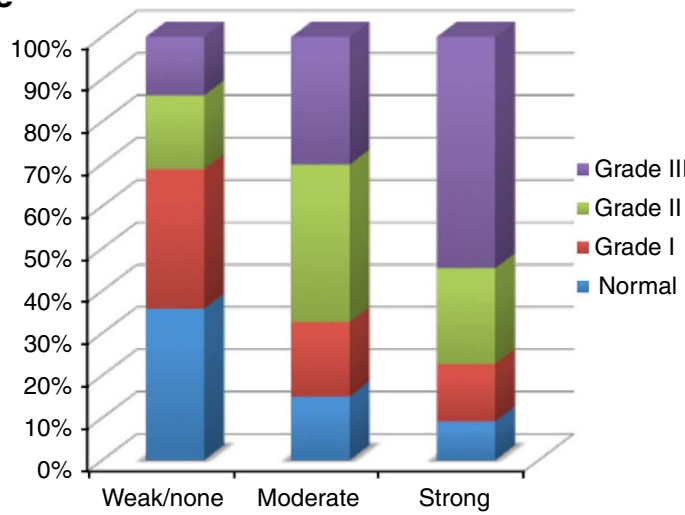

b

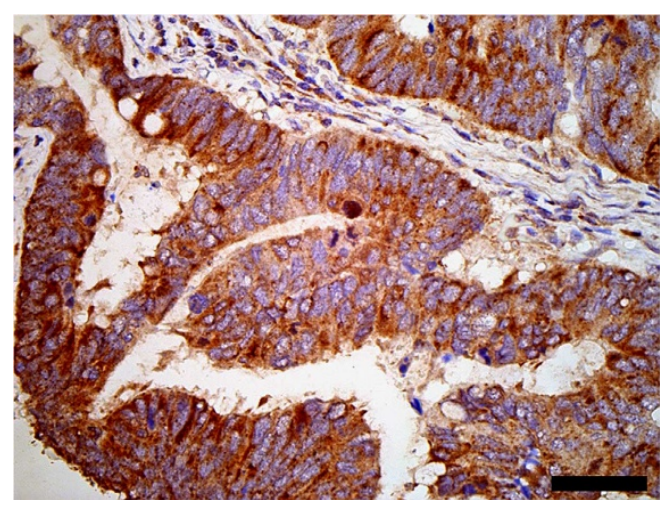

d

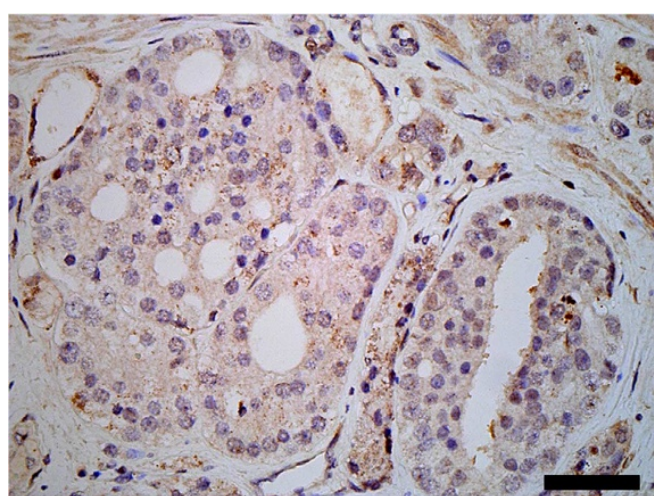

f

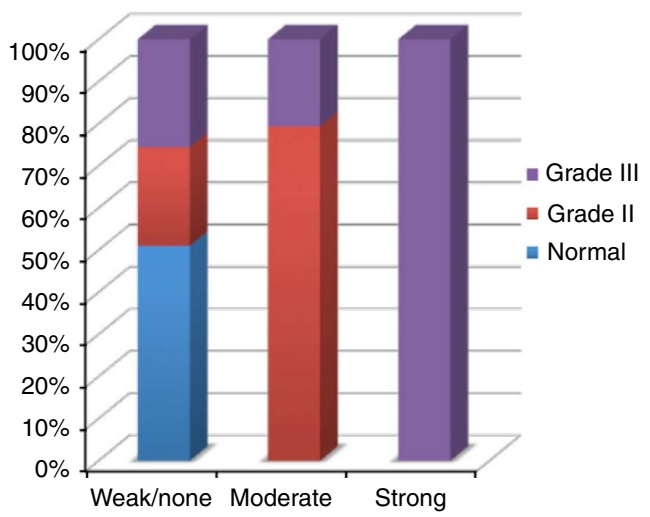

Figure 5 | Analysis of human colon and prostate cancer tissues for Y160 phosphorylation. Using the pY160 antibody IHC was performed on multi-tumours tissue microarray of 95 samples with 40 types of tumours from 27 organs. (a) Weak or no staining for Grb2 Y160 phosphorylation in grade I colon adenocarcinoma. (b) Strong staining for Grb2 Y160 phosphorylation in grade III colon adenocarcinoma. (c) Weak or no staining for Grb2 Y160 phosphorylation in grade II prostate adenocarcinoma. (d) Moderate staining for Grb2 Y160 phosphorylation in grade III prostate adenocarcinoma. A significant increase in the level of pY160 phosphorylation is seen in higher-grade tumour samples. The bars on a-d correspond to $50 \mu \mathrm{m}$. (e) The pY160 antibody staining patterns for 118 colon cancer tissue samples. The samples were scored according to the pY160 staining as weak or none, moderate and strong and plotted against tumour grade as percentage. Data compiled from normal tissues $(n=42)$, and tumours grade I ( $n=28)$, grade II $(n=34)$ and grade III $(n=14)$ which shows a progressive increase in the strength of pY160 staining with higher tumour grade. (f) The pY160 antibody staining patterns for 42 clinical prostate cancer tissue samples with the relative staining patterns for pY160 antibody. As above, samples were scored, sorted and plotted against tumour grade as percentage. Normal and/or hyperplasic tissue $(n=15)$, tumour grade II $(n=13)$ and grade III $(n=14)$. Here the pY160 staining is only associated with malignant tumours and intensity of staining is increased in tumours with a higher level of malignancy.

Cell imaging and FLIM. Cells were co-transfected with fluorescent protein-tagged Grb2 and then grown on glass coverslips for $24 \mathrm{~h}$ before experimentation. As controls, cells expressing CFP alone or CFP-WT $\mathrm{Grb} 2$ with RFP-alone were also analysed. All experiments were carried out in the presence of serum unless indicated. Cells were fixed by addition of $4 \%$ (w/vol) paraformaldehyde, $\mathrm{pH}$ 8.0. Cells were washed six or seven times with PBS, $\mathrm{pH}$ 8.0, and then mounted onto a slide with mounting medium $(0.1 \%$ p-phenylenediamine $/ 75 \%$ glycerol in PBS at $\mathrm{pH}$ 7.5-8.0). Cells were imaged using a Leica SP5 II confocal microscope with internal PMT FLIM detector. CFP was excited at $860 \mathrm{~nm}$ with titanium-sapphire pumped laser (Mai Tai BB, Spectral Physics) and SPC830 data and image acquisition card for time-correlated single photon counting. Data processing and analysis were done using B\&H SPC FLIM analysis software. The fluorescence decays were fit with a single exponential decay model. FLIM experiments were performed as described previously ${ }^{20,28,29,33}$.

Single molecule FLIM. For Grb2 as FRET donor histidine-tagged Grb2 was bacterially expressed and purified, labelled with Atto488 NHS ester according to 
the manufacturers' instructions. For Grb2 as FRET acceptor, histidine-tagged Grb2 was expressed and purified and the tag was removed by TEV cleavage. Uncleaved proteins and TEV were removed by subtractive gel filtration. Purified Grb2 was then labelled with Atto 550 NHS ester according to the manufacturer instructions. In both cases free dyes were removed by size exclusion chromatography and quantified. Only labelled proteins with 1:1 (protein:dye) ratio was used for experiments.

Atto488-Grb2 was loaded onto Talon beads and placed on a 35-mm glassbottom dish (MatTek Corporation) containing $250 \mu \mathrm{l}$ PBS pH 7.5. Beads were allowed to settle and then the reference lifetime of the Atto488-Grb2 in the absence of acceptor was measured. Without disturbing the beads a final concentration $0.5 \mu \mathrm{M}$ Atto550-Grb2 was then added and allowed to equilibrate and form dimers for 30-60 min. The lifetime was again measured and proceeded to the next treatment. For each treatment extreme care was taken to avoid disturbing the beads. For smFLIM with GFP-tagged Grb2, GFP-Grb2, was immobilized on GFP-trap agarose beads (Chromotek).

Immunohistochemistry staining. All tissues utilized in this study were of human origin and purchased from Pantomics Inc and employed in IHC experiments and scoring according to research protocols approved by the IACUC of The University of Texas MD Anderson Cancer Center (Houston, TX). Slides were baked at $60^{\circ} \mathrm{C}$ for $30 \mathrm{~min}$ and then the temperature was increased to $72^{\circ} \mathrm{C}$. Slides were then rinsed three times with Bond Dewax followed by absolute alcohol and Bond Wash. The temperature was then increased to $100^{\circ} \mathrm{C}$, and Bond ER Solution $1(\mathrm{pH} 6)$ was applied and incubated for $30 \mathrm{~min}$. Slides were rinsed with Bond Wash and the temperature was allowed to come down to room temperature. Anti-phospho-pY160 antibody was applied at 1:50 dilution $\left(0.6 \mathrm{mg} \mathrm{ml}^{-1}\right.$ final $)$ and incubated for $15 \mathrm{~min}$ at room temperature followed by rinsing with Bond Wash. Post Primary was applied and incubated for $8 \mathrm{~min}$, then slides were again rinsed with Bond Wash. Bond Polymer was applied and incubated for 8 min followed by rinsing with Bond Wash two times and then with distilled water. Peroxide Block was applied and incubated for $5 \mathrm{~min}$, then rinsed with Bond Wash four times and then washed with distilled water. $3,3^{\prime}$-Diaminobenzidine $(\mathrm{DAB})$ was applied and incubated for $10 \mathrm{~min}$ then rinsed with distilled water three times. Haematoxylin was applied and incubated for $7 \mathrm{~min}$, then rinsed with Bond Wash. Slides were then removed from Leica Bond III and rinsed with distilled water four times. They were then dehydrated in graded alcohols and xylene, then coverslipped with ClearMount from American MasterTech using Leica CV5030 coverslipper. Preparation of IHC slides for cultured cells, $5 \times 150 \mathrm{~mm}$ dishes were used per block. Cells were scraped from dishes with a rubber policeman and pelleted by centrifugation at $800 \mathrm{rpm}$ for $5 \mathrm{~min}$. The cell pellet was fixed overnight with $10 \%$ formalin and then changed to $70 \%$ ethanol before embedding into formalin fixed paraffin embedded (FFPE) blocks.

Microscale thermophoresis. The MST method has been described in detail elsewhere $^{34}$. The KD, dimer for Grb2 dimers was measured using the Monolith NT.115 from Nanotemper Technologies. Proteins were fluorescently labelled with Atto488 according to the manufacturer's protocol. Labelling efficiency was determined to be 1:1 (protein to dye) by measuring the absorbance at 280 and $488 \mathrm{~nm}$. A solution of unlabelled Grb2 was serially diluted from about $30 \mu \mathrm{M}$ to $7.3 \mathrm{nM}$ in the presence of $100 \mathrm{nM}$ labelled Grb2. The samples were loaded into silica capillaries (Polymicro Technologies) after incubation at room temperature for $15 \mathrm{~min}$. Measurements were performed at $20^{\circ} \mathrm{C}$ in PBS without calcium and magnesium (Lonza) containing $0.01 \%$ Tween 20 , by using $20 \%$ LED power and $40 \%$ IR-laser power. Measurements were also carried out on 40 and 60\% IR-Laser power for comparison. Data analyses were performed using Nanotemper Analysis software, v.1.2.101.

\section{References}

1. Rozakis-Adcock, M. et al. Association of the Shc and Grb2/Sem5 SH2containing proteins is implicated in activation of the Ras pathway by tyrosine kinases. Nature 360, 689-692 (1992).

2. Cheng, S. Y., Sun, G., Schlaepfer, D. D. \& Pallen, C. J. Grb2 promotes integrininduced focal adhesion kinase (FAK) autophosphorylation and directs the phosphorylation of protein tyrosine phosphatase alpha by the Src-FAK kinase complex. Mol. Cell. Biol. 34, 348-361 (2014).

3. Chu, J., Liu, Y., Koretzky, G. A. \& Durden, D. L. SLP-76-Cbl-Grb2-Shc interactions in FcgammaRI signaling. Blood 92, 1697-1706 (1998).

4. Myers, Jr. M. G. et al. Role of IRS-1-GRB-2 complexes in insulin signaling. Mol. Cell. Biol. 14, 3577-3587 (1994).

5. Ong, S. H., Dilworth, S., Hauck-Schmalenberger, I., Pawson, T. \& Kiefer, F. ShcA and Grb2 mediate polyoma middle T antigen-induced endothelial transformation and Gab1 tyrosine phosphorylation. EMBO J. 20, 6327-6336 (2001).

6. Ong, S. H. et al. FRS2 proteins recruit intracellular signaling pathways by binding to diverse targets on fibroblast growth factor and nerve growth factor receptors. Mol. Cell. Biol. 20, 979-989 (2000).

7. Zhou, L., Talebian, A. \& Meakin, S. O. The signaling adapter, FRS2, facilitates neuronal branching in primary cortical neurons via both Grb2- and Shp2-dependent mechanisms. J. Mol. Neurosci. 55, 663-677 (2014).
8. Chardin, P. et al. Human Sos1: a guanine nucleotide exchange factor for Ras that binds to GRB2. Science 260, 1338-1343 (1993).

9. Skolnik, E. Y. et al. The SH2/SH3 domain-containing protein GRB2 interacts with tyrosine-phosphorylated IRS1 and Shc: implications for insulin control of ras signalling. EMBO J. 12, 1929-1936 (1993).

10. Buday, L. \& Downward, J Epidermal growth factor regulates p21ras through the formation of a complex of receptor, Grb2 adapter protein, and Sos nucleotide exchange factor. Cell 73, 611-620 (1993).

11. Gale, N. W., Kaplan, S., Lowenstein, E. J., Schlessinger, J. \& Bar-Sagi, D. Grb2 mediates the EGF-dependent activation of guanine nucleotide exchange on Ras. Nature 363, 88-92 (1993).

12. Lin, C. C. et al. Inhibition of basal FGF receptor signaling by dimeric Grb2. Cell 149, 1514-1524 (2012)

13. Maignan, S. et al. Crystal structure of the mammalian Grb2 adaptor. Science 268, 291-293 (1995).

14. McDonald, C. B., Seldeen, K. L., Deegan, B. J. \& Farooq, A. Structural basis of the differential binding of the SH3 domains of Grb2 adaptor to the guanine nucleotide exchange factor Sos1. Arch. Biochem. Biophys. 479, 52-62 (2008).

15. Ghosh, J. \& Miller, R. A. Rapid tyrosine phosphorylation of Grb2 and Shc in $\mathrm{T}$ cells exposed to anti-CD3, anti-CD4, and anti-CD45 stimuli: differential effects of aging. Mech. Ageing. Dev. 80, 171-187 (1995).

16. Haines, E. et al. Tyrosine phosphorylation of Grb2: role in prolactin/epidermal growth factor cross talk in mammary epithelial cell growth and differentiation. Mol. Cell. Biol. 29, 2505-2520 (2009).

17. Anselmi, F. et al. c-ABL modulates MAP kinases activation downstream of VEGFR-2 signaling by direct phosphorylation of the adaptor proteins GRB2 and NCK1. Angiogenesis 15, 187-197 (2012).

18. Jones, D. A. \& Benjamin, C. W. Phosphorylation of growth factor receptor binding protein-2 by pp60c-src tyrosine kinase. Arch. Biochem. Biophys. 337, 143-148 (1997).

19. Li, S., Couvillon, A. D., Brasher, B. B. \& Van Etten, R. A. Tyrosine phosphorylation of Grb2 by $\mathrm{Bcr} / \mathrm{Abl}$ and epidermal growth factor receptor: a novel regulatory mechanism for tyrosine kinase signaling. $E M B O ~ J .20$, 6793-6804 (2001).

20. Ahmed, Z. et al. Grb2 controls phosphorylation of FGFR2 by inhibiting receptor kinase and Shp2 phosphatase activity. J. Cell Biol. 200, 493-504 (2013).

21. Riera, L. et al. Involvement of Grb2 adaptor protein in nucleophosminanaplastic lymphoma kinase (NPM-ALK)-mediated signaling and anaplastic large cell lymphoma growth. J. Biol. Chem. 285, 26441-26450 (2010).

22. Yuzawa, S. et al. Solution structure of Grb2 reveals extensive flexibility necessary for target recognition. J. Mol. Biol. 306, 527-537 (2001).

23. Fincham, V. J., James, M., Frame, M. C. \& Winder, S. J. Active ERK/MAP kinase is targeted to newly forming cell-matrix adhesions by integrin engagement and v-Src. EMBO J. 19, 2911-2923 (2000).

24. Huang, C., Jacobson, K. \& Schaller, M. D. MAP kinases and cell migration. J. Cell. Sci. 117, 4619-4628 (2004).

25. Pan, C. Q., Liou, Y. C. \& Low, B. C. Active Mek2 as a regulatory scaffold that promotes Pin1 binding to BPGAP1 to suppress BPGAP1-induced acute Erk activation and cell migration. J. Cell. Sci. 123, 903-916 (2010).

26. Lowenstein, E. J. et al. The SH2 and SH3 domain-containing protein GRB2 links receptor tyrosine kinases to ras signaling. Cell 70, 431-442 (1992).

27. Matuoka, K., Shibata, M., Yamakawa, A. \& Takenawa, T. Cloning of ASH, a ubiquitous protein composed of one Src homology region (SH) 2 and two SH3 domains, from human and rat cDNA libraries. Proc. Natl Acad. Sci. USA 89, 9015-9019 (1992).

28. Ahmed, Z. et al. Direct binding of Grb2 SH3 domain to FGFR2 regulates SHP2 function. Cell Signal. 22, 23-33 (2010).

29. Ahmed, Z., Schuller, A. C., Suhling, K., Tregidgo, C. \& Ladbury, J. E. Extracellular point mutations in FGFR2 elicit unexpected changes in intracellular signalling. Biochem. J. 413, 37-49 (2008).

30. Schuller, A. C., Ahmed, Z. \& Ladbury, J. E. Extracellular point mutations in FGFR2 result in elevated ERK1/2 activation and perturbation of neuronal differentiation. Biochem. J. 410, 205-211 (2008).

31. Schuller, A. C. et al. Indirect recruitment of the signalling adaptor She to the fibroblast growth factor receptor 2 (FGFR2). Biochem. J. 416, 189-199 (2008).

32. Suen, K. M. et al. Interaction with Shc prevents aberrant Erk activation in the absence of extracellular stimuli. Nat. Struct. Mol. Biol. 20, 620-627 (2013).

33. George, R. et al. A complex of Shc and Ran-GTPase localises to the cell nucleus. Cell. Mol. Life. Sci. 66, 711-720 (2009).

34. Seidel, S. A. I. et al. Microscale thermophoresis quantifies biomolecular interactions under previously challenging conditions. Methods 59, 301-315 (2013).

\section{Acknowledgements}

We thank PicoQuant GmbH for the time-correlated anisotropy measurements, analysis and advice. We acknowledge Shilpa Muralidhar for technical assistance. 


\section{Author contributions}

Z.A. devised and performed experiments and wrote manuscript. Z.T. performed cell and tissue biology experiments. K.M.S. provided cloning and C.-C. L. and G.R.L. protein preparation expertise. N.P.C. and A.A.M. performed fluorescence spectroscopic experiments. M.G. contributed pathology analysis. J.E.L. devised experiments and wrote manuscript.

\section{Additional information}

Supplementary Information accompanies this paper at http://www.nature.com/ naturecommunications

Competing financial interests: The authors declare no competing financial interests.
Reprints and permission information is available online at http://npg.nature.com/ reprintsandpermissions/

How to cite this article: Ahmed, Z. et al. Grb2 monomer-dimer equilibrium determines normal versus oncogenic function. Nat. Commun. 6:7354 doi: 10.1038/ncomms8354 (2015).

(c) (i) This work is licensed under a Creative Commons Attribution 4.0 International License. The images or other third party material in this article are included in the article's Creative Commons license, unless indicated otherwise in the credit line; if the material is not included under the Creative Commons license, users will need to obtain permission from the license holder to reproduce the material. To view a copy of this license, visit http://creativecommons.org/licenses/by/4.0/ 


\section{Corrigendum: Grb2 monomer-dimer equilibrium determines normal versus oncogenic function}

Zamal Ahmed, Zahra Timsah, Kin M. Suen, Nathan P. Cook, Gilbert R. Lee IV, Chi-Chuan Lin, Mihai Gagea, Angel A. Marti \& John E. Ladbury

Nature Communications 6:7354 doi: 10.1038/ncomms8354 (2015); Published 24 Jun 2015; Updated 3 Aug 2015.

In this Article, there are errors in the labelling of the $y$ axes in Figs 2 and 3. In both figures, 'Fluorescence intensity' should read 'Number of pixels'. The correct versions of these figures appear below.

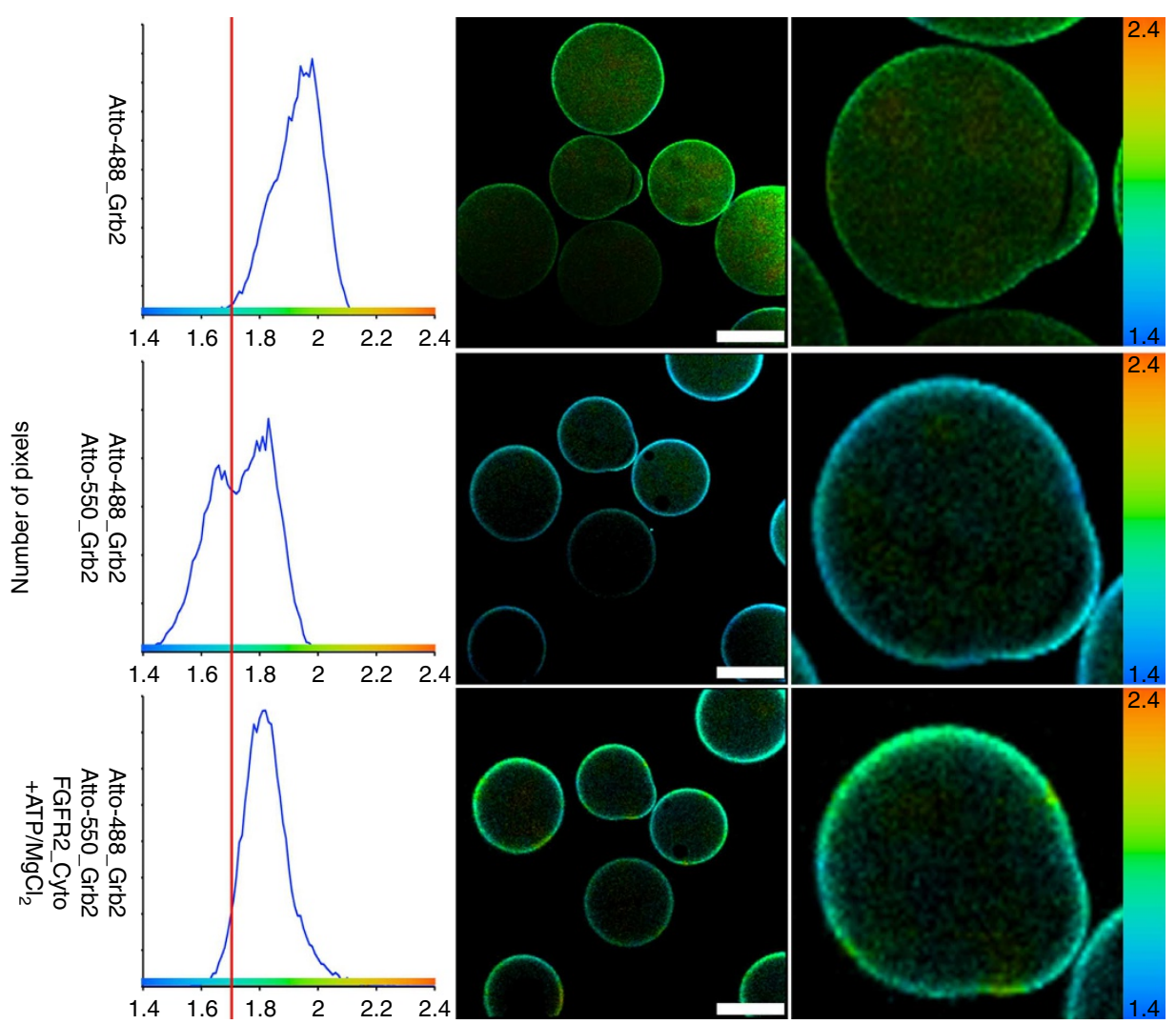

Figure 2 


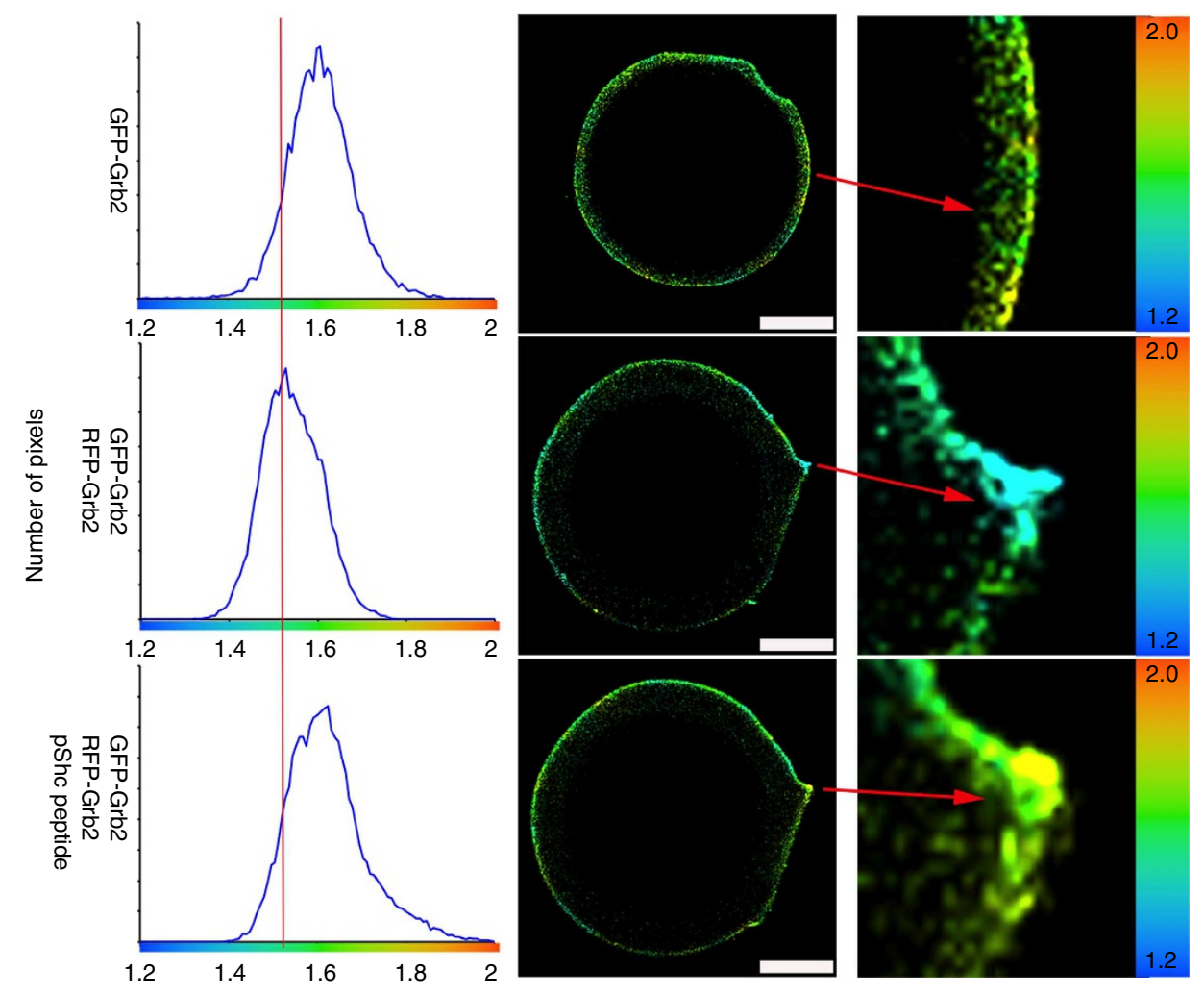

Figure 3 